\title{
SUBPRODUCTOS DE PAPA EN LA ALIMENTACIÓN ESTRATÉGICA DE OVINOS DURANTE LA ESTACIÓN SECAEN EL VALLE DELMANTARO
}

\author{
Potato By-Products for Sheep Strategic Feeding during the Dry Season in \\ THE Mantaro VaLley
}
Ronald Jiménez A., ${ }^{1,2}$, Felipe San Martín H. ${ }^{3}$, Héctor Huamán U. ${ }^{3}$, Miguel Ara G. ${ }^{3}$, Amparo Huamán C. ${ }^{1}$

\section{RESUMIEN}

Se evaluó el efecto de niveles crecientes de suplementación con subproducto de papa sobre la ganancia de peso vivo en ovinos. Se usó 120 ovinos Junín (69 hembras y 51 machos) en un arreglo factorial de 5 tratamientos $x 2$ sexos. Un tratamiento fue de solo pastoreo por 8 h y los otros cuatro fueron de 4 h/día de pastoreo más rastrojo de maíz amonificado (550 g/animal(día) con adición de 0, 200, 400 y 600 g/ovino/día de subproducto de papa para un periodo de engorde de 24 días. La respuesta en ganancia de peso a la suplementación con subproducto de papa fue mayor para ovinos machos $(\mathrm{p}<0.01)$, en los cuales el patrón de respuesta para los niveles crecientes de subproducto de papa fue de tipo cuadrático $(\mathrm{p}<0.05)$, y donde un nivel de 570 g de subproducto de papa dio ganancias de peso e índices económicos similares a los del sistema pastoril ( 8 h/día). Se concluye que la inclusión de subproducto de papa en niveles de 570 g/ovino/día a dietas de pastoreo restringido (4 h/día) más rastrojo de maíz amonificado es una estrategia de alimentación viable y económica para engordar ovinos machos durante la estación seca en la Sierra peruana.

Palabras clave: ovino, subproducto de papa, rastrojo de maíz amonificado, suplementación, engorde

\section{Abstract}

The effect of increasing levels of supplementation with potato by-products on sheep body weight gain was evaluated. It was used 120 Junín sheep (69 female and 51 male) in a 2 x 5 factorial arrangement (two sexes x 5 feeding types). One treatment was $8 \mathrm{~h} /$ day of grazing and other four with addition of 0, 200, 400 and $600 \mathrm{~g}$ of potato by-products over ammoniated corn crop residue basal diet on 4-h a day grazing system for 24 days. Body weight gain was higher in males $(\mathrm{p}<0.01)$ in which the response pattern to the increasing

\footnotetext{
${ }^{1}$ Estación Experimental El Mantaro, Instituto Veterinario de Investigaciones Tropicales y de Altura (IVITA), Junín, ${ }^{3}$ Laboratorio de Bioquímica, Nutrición y Alimentación Animal, Facultad de Medicina Veterinaria, Universidad Nacional Mayor de San Marcos, Lima

${ }^{2}$ E-mail: rjimeneza@unmsm.edu.pe
} 
levels of potato by-products supplementation was quadratic $(p<0.01)$, and the level of $570 \mathrm{~g}$ of potato by-products gave a biological and economic performance similar to the full (8-h a day) grazing system. Data suggested that the inclusion of $570 \mathrm{~g}$ of potato byproducts on ammoniated corn crop residues is a practical and economical feeding strategy for fattening male sheep on a 4-h a day restricted grazing regime during the dry season in the Peruvian highlands.

Keywords: sheep, potatoes by-products, ammoniated corn crop residues, supplementation, fattening

\section{INTRODUCCIÓN}

La cantidad de materia seca producida por los pastos cultivados durante la estación seca en la Sierra Central del Perú representa el $40 \%$ de lo producido por estas mismas pasturas en la estación lluviosa (Bojórquez, 1998). Estas diferencias conllevan a utilizar menores cargas animales para el pastoreo en la estación seca.

Con el fin de evitar una merma en la productividad animal durante la época seca, algunos criadores optan por complementar la alimentación con rastrojos de cosecha, pero el aporte nutritivo es menor. La amonificación mejora el aporte de nitrógeno, la digestibilidad y el consumo de los rastrojos (Horton y Steacy, 1979; Badurdeen et al., 1994), pero no aporta los insumos necesarios, principalmente carbohidratos fermentables, para la producción eficiente de nutrientes a nivel ruminal $\mathrm{y}$, por tanto, no satisface la demanda de nutrientes de rumiantes en etapas productivas. La fermentación ruminal puede mejorarse suplementando carbohidratos fácilmente degradables, como los contenidos en los subproductos de tubérculo de papa (productos no aptos para comercio ni consumo), y de esa manera, facilitando la producción de proteína microbiana, mientras no se altere la ecología ruminal.

En el valle del Mantaro predomina el cultivo de papa y se estima una producción de 36000 TM anuales de subproducto de papa
(INEI, 1996). Actualmente, el desperdicio de tubérculo de papa, se emplea en la alimentación de cerdos y bovinos, pero en forma empírica. El presente estudio busca generar información sobre el uso eficiente del subproducto de tubérculo de papa, al incorporarlo como suplemento energético estratégico en la alimentación de ovinos durante la estación seca.

\section{Materiales y Métodos}

El estudio se realizó entre julio a setiembre de 2006 en la Estación Experimental del Centro de Investigación IVITA-El Mantaro, Junín, a una altitud de 3320 msnm. La zona tiene una temperatura ambiental promedio que oscila entre 19.9 y $-1.8^{\circ} \mathrm{C}$ y una precipitación pluvial anual de $770 \mathrm{~mm}$.

Se emplearon 120 ovinos Junín de 7 meses de edad (69 hembras y 51 machos), distribuidos al azar en un diseño factorial de 5 tratamientos x 2 sexos. En el tratamiento control (T0) los ovinos se mantuvieron en pastoreo por 8 horas (08:00 a 16:00), mientras que en los tratamientos $\mathrm{T} 1$ a $\mathrm{T} 4$ estuvieron en pastoreo por 4 horas (12:00 a 16:00), y recibieron $550 \mathrm{~g} /$ animal/día de rastrojo de maíz amonificado, además de 0 (T1), 200 (T2), 400 (T3) y 600 g/día (T4) de subproducto de papa picada. La evaluación nutricional de los insumos se muestra en el Cuadro 1. 
Cuadro 1. Análisis químico proximal de los insumos alimenticios utilizados en el experimento

\begin{tabular}{lccccccc}
\hline & Materia & & \multicolumn{5}{c}{ Contenido (\%) de nutrientes en base seca } \\
\cline { 5 - 8 } Insumo & $\begin{array}{c}\text { seca } \\
\text { (\%) }\end{array}$ & $\begin{array}{c}\text { Humedad } \\
(\%)\end{array}$ & $\begin{array}{c}\text { Proteína } \\
\text { cruda }\end{array}$ & $\begin{array}{c}\text { Fibra } \\
\text { cruda }\end{array}$ & Cenizas & $\begin{array}{c}\text { Extracto } \\
\text { etéreo }\end{array}$ & $\begin{array}{c}\text { Extracto } \\
\text { libre de } \\
\text { nitrógeno }\end{array}$ \\
\hline $\begin{array}{l}\text { Pastura } \\
\text { asociada }\end{array}$ & 25 & 75 & 14.6 & 36.0 & 7.1 & 2.2 & 40.1 \\
$\begin{array}{l}\text { Rastrojo } \\
\text { amonificado }\end{array}$ & 65 & 35 & 14.0 & 29.0 & 6.6 & 0.9 & 49.5 \\
$\begin{array}{l}\text { Subproducto } \\
\text { de papa }\end{array}$ & 39 & 61 & 8.6 & 4.3 & 5.0 & 0.6 & 81.5 \\
\hline
\end{tabular}

Los animales fueron desparasitados antes del experimento e identificados con pintura de acuerdo al grupo de tratamiento. El pastoreo se hizo en forma conjunta en una pastura compuesta por rye grass italiano de variedades Boxer y Tama, más trébol rojo de la variedad Quinequelli. Se empleó una carga de 30 ovinos por hectárea por año. Se tuvo una fase de acostumbramiento al rastrojo amonificado y al subproducto de papa de ocho días.

Se empleó 2.7 TM de rastrojo de maíz, picado mecánicamente a un tamaño de partícula de $2.3 \mathrm{~cm}$. El rastrojo fue amonificado con una solución de urea y conservado en 19 sacos plásticos de $1 \mathrm{~m}$ de diámetro y con una altura de llenado de $1.5 \mathrm{~m}$, manteniendo una relación urea: agua: materia seca de 5:50:100. Los sacos fueron compactados y sellados y el forraje amonificado se usó a los 12 meses de su elaboración. Cuatro sacos tuvieron que ser desechados por enmohecimiento del rastrojo.

Los animales tuvieron acceso a una fuente de agua en sus corrales. El consumo del rastrojo y la ganancia de peso individual se midieron durante 24 días. En el análisis estadístico se empleó el análisis de varianza con la suma de cuadrados tipo III, sugerido para experimentos desbalanceados, para un diseño factorial con aplicación de polinomios ortogonales para los niveles de suplementación con papa, donde la variable de respuesta fue la ganancia de peso. Los análisis se realizaron con el programa SAS (SAS, 2000).

El análisis económico se realizó mediante la estimación de costos fijos y variables para el periodo de engorde evaluado. Los costos fijos fueron estimados por ovino/ día, a excepción de la pastura que fue estimada en hora de pastoreo/ovino, con la finalidad de dar mayor precisión al costeo por tratamientos. Los costos variables empleados para el cálculo económico de cada tratamiento corresponden a los costos de los insumos alimenticios empleados. El costo de producción fue estimado por la suma de los costos fijos y variables.

Para la estimación del beneficio, se asumió que un kilogramo de peso vivo de ovino cuesta S/. 3.50. El beneficio final se obtuvo al multiplicar el peso ganado durante el ensayo según cada tratamiento por el costo del kilogramo de ovino vivo. 
Cuadro 2. Efecto de la suplementación con rastrojo de maíz amonificado (RMA) y subproducto de papa fresca (SPF) sobre la ganancia de peso de ovinos bajo pastoreo (g/ovino/día) durante un periodo de 24 días

\begin{tabular}{lcc}
\hline Tratamiento & Macho & Hembra \\
\hline T0: Pastoreo 8 h & 202 & 131 \\
T1: Pastoreo 4 h + RMA & 177 & 106 \\
T2: Pastoreo 4 h + RMA + 200 g de SPF & 146 & 100 \\
T3: Pastoreo 4 h + RMA + 400 g de SPF & 144 & 109 \\
T4: Pastoreo 4 h + RMA + 600 g de SPF & 203 & 109 \\
\hline
\end{tabular}

Cuadro 3. Consumos parciales de rastrojo amonificado de maíz con niveles crecientes de subproducto de papa en ovinos bajo pastoreo en pastura cultivada durante 24 días

\begin{tabular}{lcccc}
\hline & T1 & T2 & T3 & T4 \\
\hline Consumo (g) MS/kg peso metabólico & & & & \\
$\quad$ Rastrojo amonificado & 23.18 & 22.97 & 21.84 & 21.67 \\
$\quad$ Subproducto de papa & 0.00 & 3.50 & 6.83 & 9.95 \\
$\quad \begin{array}{l}\text { Rastrojo amonificado y } \\
\text { subproducto de papa }\end{array}$ & 23.18 & 26.46 & 28.67 & 31.63 \\
$\quad$ & & & & \\
Consumo porcentual del peso vivo & 0.96 & 0.97 & 0.91 & 0.90 \\
$\quad \begin{array}{l}\text { Rastrojo amonificado } \\
\text { Subproducto de papa }\end{array}$ & 0.00 & 0.15 & 0.29 & 0.41 \\
$\quad \begin{array}{l}\text { Rastrojo amonificado y } \\
\text { subproducto de papa }\end{array}$ & 0.96 & 1.12 & 1.20 & 1.31 \\
\hline 1 T0: Pastoreo 8 h, T1: Pastoreo 4 h + RMA, T2: Pastoreo 4 h + RMA + 200 g de SPF, T3: Pastoreo 4 \\
h + RMA + 400 g de SPF, T4: Pastoreo 4 h + RMA + 600 g de SPF & &
\end{tabular}

\section{Resultados y Discusión}

Los sistemas pastoriles de climas templados basados en asociaciones gramínea leguminosa, como los que existen en la Sierra Central, resultan suficientes para lograr cubrir satisfactoriamente las necesidades nutritivas de los ovinos en crecimiento cuando la disponibilidad del forraje no resulta restrictivo, para que los ovinos puedan maximizar su selección e ingerir los componentes foliares de mayor digestibilidad (Preston y Leng, 1987).
Los resultados indicaron una superioridad en la ganancia de peso de ovinos en el sistema pastoril (T0) frente a la mayoría de los demás tratamientos (Cuadro 2). Se encontró una respuesta de tendencia cuadrática $(p<0.05)$ cuando se aplicaron niveles crecientes de suplementación con subproducto de papa a raciones que combinaban cuatro horas de pastoreo más rastrojo de maíz amonificado. El mismo patrón de respuesta cuadrática se reporta en bovinos de carne suplementados con niveles crecientes de subproducto de papa (Radunz et al., 2003). 
Cuadro 4. Indicadores económicos para sistemas de alimentación en el engorde de ovinos para un periodo de 24 días empleando rastrojo de maíz amonificado (RMA) y subproducto de papa fresco (SPF)

\begin{tabular}{lcccccc}
\hline \multirow{3}{*}{ Sexo } & Tratamiento $^{1}$ & $\begin{array}{c}\text { Costo de } \\
\text { producción } \\
(\text { S/.) }\end{array}$ & $\begin{array}{c}\text { Ganancia } \\
\text { de peso } \\
\text { (kg) }\end{array}$ & Beneficio & $\begin{array}{c}\text { Ratio } \\
\text { B/C }\end{array}$ & $\begin{array}{c}\text { Rentabilidad } \\
(\%)\end{array}$ \\
\hline \multirow{5}{*}{ Macho } & T0 & 12.1 & 4.8 & 17.0 & 1.4 & 40 \\
& T1 & 11.2 & 4.2 & 14.9 & 1.3 & 33 \\
& T2 & 11.4 & 3.5 & 12.3 & 1.1 & 8 \\
& T3 & 11.6 & 3.5 & 12.1 & 1.0 & 5 \\
& T4 & 11.8 & 4.9 & 17.1 & 1.4 & 44 \\
& T0 & 12.1 & 3.1 & 10.9 & 0.9 & -10 \\
& T1 & 11.2 & 2.5 & 8.8 & 0.8 & -22 \\
& T2 & 11.4 & 2.4 & 8.4 & 0.7 & -26 \\
& T3 & 11.6 & 2.6 & 9.1 & 0.8 & -23 \\
& T4 & 11.8 & 2.6 & 9.1 & 0.8 & -23 \\
\hline \multirow{6}{*}{ Prombra } & T0 & 12.1 & 4.0 & 13.9 & 1.1 & 15 \\
& T1 & 11.2 & 3.4 & 11.8 & 1.1 & 5 \\
& T2 & 11.4 & 3.0 & 10.3 & 0.9 & -9 \\
& T3 & 11.6 & 3.0 & 10.4 & 0.9 & -10 \\
& T4 & 11.8 & 3.7 & 13.1 & 1.1 & 11 \\
\hline
\end{tabular}

${ }^{1}$ T0: Pastoreo 8 h, T1: Pastoreo 4 h + RMA, T2: Pastoreo 4 h + RMA + 200 g de SPF, T3: Pastoreo 4 $\mathrm{h}+\mathrm{RMA}+400 \mathrm{~g}$ de SPF, T4: Pastoreo $4 \mathrm{~h}+\mathrm{RMA}+600 \mathrm{~g}$ de SPF

El reemplazo de cuatro horas de pastoreo con rastrojo de maíz amonificado resultó en menor ganancia de peso, posiblemente debido a que este producto tiende a reducir la tasa de pasaje y consecuentemente el consumo, respecto a los niveles de consumo que se reportan para ovinos en pastoreo de pasturas cultivadas (3.6\% del peso vivo en MS para una pastura que produce $12 \mathrm{TM} / \mathrm{ha} / \mathrm{año} \mathrm{y} \mathrm{to-}$ lera una carga de 30 ovinos con $30 \mathrm{~kg}$ de peso). Además, es posible que el nitrógeno proporcionado por el rastrojo amonificado genere un desbalance entre proteína y energía en la ración, con la consecuente ineficiencia en la síntesis de proteína microbiana y pérdidas de energía, dando lugar a una menor respuesta en la ganancia de peso.
Se puede comprobar un ligero descenso en el consumo de materia seca del rastrojo cuando se incrementa el nivel de subproducto de papa, pero el consumo acumulado parcial aumenta (Cuadro 3), indicando un efecto aditivo y sustitutivo de la papa sobre el rastrojo. Es de esperarse que los consumos de pastos cultivados en las cuatro horas de pastoreo fueran decrecientes e inversamente proporcionales a los niveles de subproducto de papa.

La menor ganancia de peso de los tratamientos T2 y T3 en relación a T1 fue inesperada, dado que estos animales recibieron el subproducto de papa. Una posible explicación sería que el almidón de la papa, clasifi- 
cado como almidón de lenta degradación (5\%/ h) puede elevar la concentración de ácidos grasos volátiles y el $\mathrm{pH}$ a nivel ruminal (Monteils et al., 2002), modificando el medio de manera sostenida por más de 12 horas para beneficio de los microorganismos amilolíticos y perjuicio de los celulolíticos, lo cual afectaría la digestibilidad y consumo del rastrojo y de la pastura. Por otro lado, se han identificado la presencia de glicoalcaloides en la papa, los que aumentan con la exposición a la luz, tornando la papa de color verdoso, $y$ con efectos tóxicos que llegan a causar la muerte celular (Friedman, 2006), incluyendo los microorganismos ruminales. Es así, que la coloración verdosa del subproducto de papa hace suponer que contenía un nivel intermedio de estos compuestos.

En el caso de T4, donde el nivel de suplementación del subproducto de papa es mayor, no modifica lo señalado para T2 y T3, pero se permitiría una mejor producción de ácido propiónico, principal precursor glucogénico, ideal para cubrir los gastos energéticos del incremento de peso. Asimismo, los efectos de los glicoalcaloides serían reducidos con el calor de la fermentación ruminal, incrementado por la inclusión de una mayor cantidad de papa. Además, el nitrógeno adicional que proporciona el rastrojo de maíz junto a la energía liberada por fermentación del subproducto de papa permitiría un equilibrio entre estos dos nutrientes, haciendo eficiente la producción de proteína microbiana, componente importante para el crecimiento de los tejidos.

Los ovinos machos mostraron una ganancia de peso superior a las hembras $(p<0.01)$, y reafirman su excelente aptitud para procesos de engorde.

Dado que los resultados de ganancia de peso muestran una tendencia cuadrática para los niveles crecientes de suplementación con subproducto de papa, se elaboraron curvas de regresión cuadrática para ovinos machos y hembras (Fig. 1). Teniendo en consideración que las mejores respuestas en ganan- cias de peso se produjeron en sistemas pastoriles sobre pasturas cultivadas con 8 horas de pastoreo (Huamán et al., 2000), las ganancias de peso máximas esperadas fueron de 202 y 131 g/día para machos y hembras, respectivamente. En base a esto, se requeriría un nivel de suplementación de 570 y $1011 \mathrm{~g}$ de subproducto de papa para ovinos machos y hembras, respectivamente, y donde el cálculo para los machos muestra una mayor confiabilidad (Fig. 1).

El suministro en conjunto del rastrojo de maíz amonificado y el subproducto de papa picado en un comedero de tipo lineal permitió observar una mayor preferencia de los ovinos por el subproducto de papa en relación al rastrojo, acción que probablemente dio lugar a ciertas ventajas para los machos en su capacidad de consumo, dado su mayor fortaleza y tamaño, respecto a las hembras. Por lo tanto, no se descarta que haya ocurrido una leve subestimación del consumo del subproducto de papa en machos; sin embargo, el nivel de suplementación recomendado no deja de ser un buen punto de referencia para su aplicabilidad en campo.

Los indicadores económicos para el presente estudio se presentan en el Cuadro 4. Resalta en primer lugar, la influencia positiva de la inclusión del rastrojo amonificado de maíz para abaratar costos y mejorar beneficios; en segundo lugar, la poca respuesta del ovino hembra para actividades de engorde, tercero, el uso de subproducto de papa solo alcanza mérito económico con altos niveles de suplementación (600g/ovino/día).

El costo promedio del rastrojo de maíz es de S/. 500.00 por hectárea, con un rendimiento promedio de $10 \mathrm{TM} / \mathrm{ha}$, pero el proceso de amonificación agrega una inversión adicional, resultando un costo aproximado de S/. 0.08/kg de MS de rastrojo de maíz amonificado. Este costo es menor que el del pasto cultivado (rye grass - trébol) estimado en $\mathrm{S} / .0 .12$, de modo que cuanto mayor sea la participación del rastrojo en la dieta beneficiará económicamente al productor. Sin 


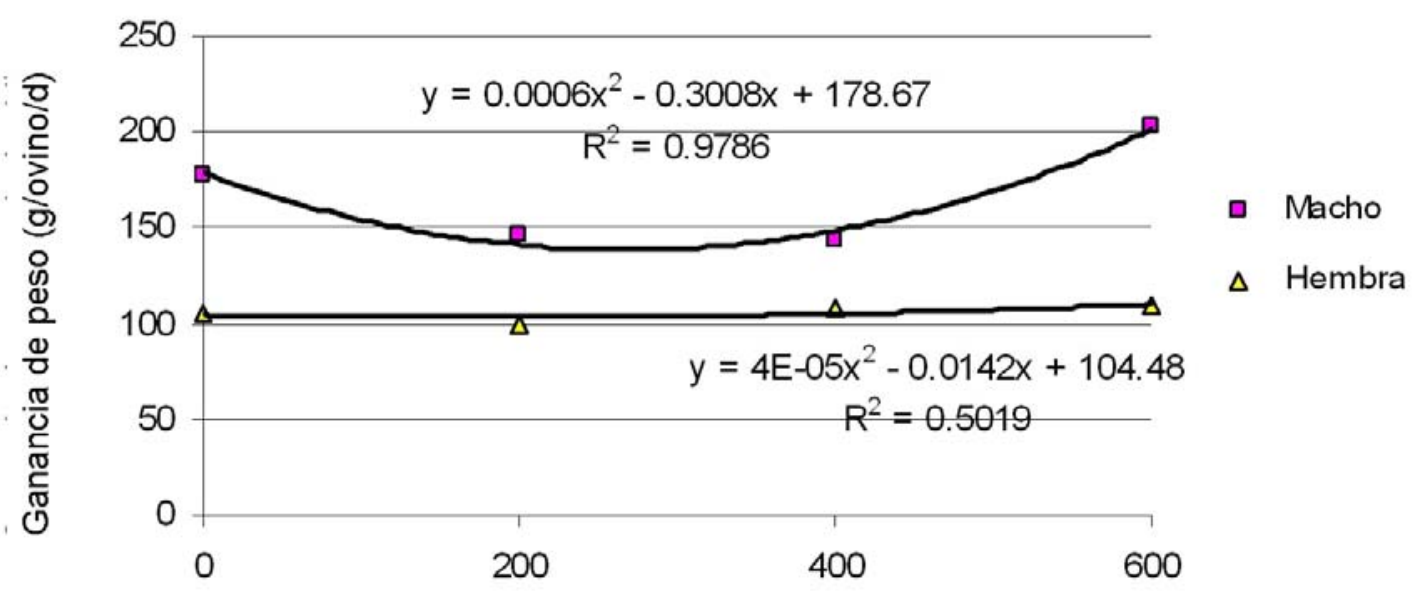

Subproducto de papa suplementada, g/ovino/dia

Figura 1. Curvas de regresión entre ganancia de peso y suplementación con subproducto de papa en ovinos bajo pastoreo en pastura cultivada durante 24 días

embargo, cuando los niveles de rastrojo de maíz amonificado alcanzan el 30\% de la ración diaria, como ocurrió en el presente estudio, la rentabilidad se reduce a causa de una menor ganancia de peso, y se esperaría un decremento marcado cuando los niveles sean mayores.

Una estrategia viable para mejorar la ganancia de peso y consecuentemente la rentabilidad de ovinos alimentados con rastrojo de maíz amonificado y pastoreo restringido en pasturas asociadas es la inclusión de insumos con alto contenido de carbohidratos solubles como la papa. Esto ocasiona un incremento en el costo de producción pero se obtiene una respuesta económica favorable cuando se emplea niveles de subproducto de papa de por lo menos 600 g/ovino/día (Cuadro 4).

\section{Conclusiones}

- La suplementación con niveles crecientes de subproducto de papa fresca a dietas de pastoreo restringido más rastrojo de maíz amonificado, muestra una res- puesta cuadrática en ganancia de peso, donde un nivel de $570 \mathrm{~g}$ de suplemento en ovinos machos permite ganancias de peso similares a las obtenidas con pastos cultivados.

- La inclusión de rastrojo de maíz amonificado en niveles no mayores a $30 \%$ en las raciones de ovinos es una alternativa viable para la estación seca, pero sus beneficios económicos no superan al sistema pastoril.

\section{LITERATURA CitadA}

1. Badurdeen AL, Ibrahim MNM, Schiere JB. 1994. Methods to improve utilization of rice straw. II. Effects of different levels of feeding on intake and digestibility of untreated and urea ammonia treated rice straw. Asian Austral J Anim 7: 165-169.

2. Bojórquez C. 1998. Producción de pastos cultivados en tres zonas agroecológicas de la Sierra Central. Rev Inv Pec 9(1): 20-31. 
3. Friedman M. 2006. Potato glycoalkaloids and metabolites: roles in the plant and in the diet. J Agric Food Chem 54: 8655-8681.

4. Horton GM, Steacy M. 1979. Effect of anhidrous ammonia treatment of the intake and digestibility of cereal straw by steers. J Anim Sci 48: 1239-1249.

5. Huamán AE, Dávalos R, San Martín F, Bojórquez C, Carcelén F, Pérez A. 2000. Compensación al menor tiempo de pastoreo con diferentes niveles de heno de alfalfa en el engorde de ovinos. Rev Inv Vet, Perú 11(2): 1-5.

6. [INEI] Instituto Nacional de Estadística e Informática. 1996. III Censo nacional agropecuario. Perfil agropecuario departamento de Junín. Lima: INEI. 400 p.
7. Monteils V, Jurjanz S, Colin-Schoellen O, Blanchart G, Laurent F. 2002. Kinetics of ruminal degradation of wheat and potato starches in total mixed rations. J Anim Sci 80: 235-241.

8. Preston TR, Leng RA. 1987. Ajustando los sistemas de producción pecuaria a los recursos disponibles: Aspectos básicos y aplicados del nuevo enfoque sobre la nutrición de rumiantes en el trópico. $2^{\mathrm{a}}$ ed. Colombia: ILCA. $312 \mathrm{p}$.

9. Radunz AE, Lardy GP, Bauer ML, Marchello MJ, Loe ER, Berg PT. 2003. Influence of steam-peeled potatoprocessing waste inclusion level in beef finishing diets: Effects on digestion, feedlot performance, and meat quality. $\mathrm{J}$ Anim Sci 81: 2675-2685.

10. SAS. 2000. SAS/STAT® User's Guide (Release 8.1). Cary, NC, USA: SAS Inst. Inc. 